“ (C) 2018 IEEE. Personal use of this material is permitted. Permission from IEEE must be obtained for all other uses, in any current or future media, including

reprinting/republishing this material for advertising or promotional purposes, creating new collective works, for resale or redistribution to servers or lists, or reuse of any copyrighted component of this work in other works." 


\title{
Analog Least Mean Square Loop for Self-Interference Cancellation in Generalized Continuous Wave SAR
}

\author{
Anh Tuyen Le ${ }^{1}$, Yijiang $\mathrm{Nan}^{1}$, Le Chung Tran ${ }^{2}$, Xiaojing Huang ${ }^{1}$, Y. Jay Guo ${ }^{1}$, and Yiannis Vardaxoglou ${ }^{3}$ \\ ${ }^{1}$ University of Technology Sydney, Ultimo, NSW, 2007, Australia \\ Email: \{anhtuyen.le, Yijiang.Nan\}estudent.uts.edu.au \\ Email: \{xiaojing.huang, jay.guo\}euts.edu.au \\ ${ }^{2}$ University of Wollongong, Wollongong, NSW, 2522, Australia \\ Email: lctran@uow.edu.au \\ ${ }^{3}$ Loughborough University, Epinal Way, Loughborough, Leicestershire, LE11 3TU, UK \\ Email: j.c.vardaxogloullboro.ac.uk
}

\begin{abstract}
Generalized continuous wave synthetic aperture radar (GCW-SAR) is a promising new imaging radar system since it applies the full-duplex (FD) transmission technique to achieve continuous signaling in order to overcome several fundamental limitations of the conventional pulsed SARs. As in any FD wireless communication system, self-interference (SI) is also a key problem which can impact on the GCW-SAR system. In this paper, the analog least mean square (ALMS) loop in the radio frequency domain is adopted to cancel the SI for a GCWSAR system with periodic chirp signaling. The average residual SI power after the ALMS loop is analyzed theoretically by a stationary analysis. It is found that the ALMS loop not only works with random signals in general FD communication systems, but also works well with the periodic signal in GCW-SAR systems. Simulation results show that over $45 \mathrm{~dB}$ SI cancellation can be achieved by the ALMS loop which ensures the proper operation of the GCW-SAR system.
\end{abstract}

Index Terms-Full-duplex, self-interference cancellation, ALMS loop, and SAR.

\section{INTRODUCTION}

The combination of a full-duplex (FD) radio technique and continuous wave $(\mathrm{CW})$ radar, called generalized continuous wave synthetic aperture radar (GCW-SAR), has been developed recently [1]. With GCW-SAR, the slow time sampling in azimuth direction used in conventional pulsed SAR and frequency modulated (FM) CW SAR systems is no longer required and thus some intrinsic limitations, such as the minimum antenna area constraint [2], can be removed. This new SAR concept enables the development of future high resolution and wide swath remote sensing technologies. However, due to the simultaneous transmitting and receiving operations, GCW-SAR faces a severe problem of self-interference (SI), which is the strong signal emitted from the transmitter blocking the receiver from detecting the signals of interest. Therefore, SI cancellation (SIC) is a key issue to be addressed for realization of GCW-SAR.

The requirement of SIC for wireless FD systems can be achieved by three levels of cancellation at propagation (or antenna), radio frequency (RF) front-end, and digital domains
[3]. However, there are two main differences between SIC in GCW-SAR systems and that in FD communication systems. Firstly, in a GCW-SAR system, a chirp signal is periodically transmitted rather than a random signal as in the communication counterpart. As a result, the behaviors of the cancellation circuits in GCW-SAR systems such as the convergence and stabilization properties may be affected. Secondly, in the GCW-SAR systems, digital domain cancellation may not be required as the residual SI does not need to be lower than the noise floor level as in the FD communication system. The reason is that the received signals in GCW-SAR can be considered as time delayed transmitted signals. The useful received signals are the far-field reflections, whereas the SI can be regarded as near field reflections which can be easily removed after image compression. Therefore, for GCW-SAR, the antenna and RF domain SIC will be of a significant importance since SIC is required to protect the analog-todigital converter (ADC) from being saturated by the strong SI. As an example, a GCW-SAR system can have a similar configuration as in a practical airborne FMCW-SAR system [4]. The transmit power is $18 \mathrm{dBm}$, and the receiver noise floor is at $-90 \mathrm{dBm}$. If the $\mathrm{ADC}$ in the receiver has the dynamic range of $60 \mathrm{~dB}$, and the signal-to-interference ratio required for a normal FD operation is $-45 \mathrm{~dB}$, the level of residual SI must be less than $-90-(-45)=-45 \mathrm{dBm}$. The SIC required can be calculated by $18-(-45)=63 \mathrm{~dB}$. Hence, about $70 \mathrm{~dB}$ of SIC must be achieved before digitalized by the ADC.

Such $70 \mathrm{~dB}$ of SIC can be achieved by two steps of SIC in the propagation domain and the RF domain. In the first step, the transmit and receive antennas are separated with a distance to attenuate the level of SI as in [5]. For example, with the operating frequency at $5 \mathrm{GHz}$, at least $25 \mathrm{~dB}$ of SI attenuation can be achieved when the transmit and receive antennas are located at a distance of $100 \mathrm{~mm}$ or higher. The rest of the required SIC can be obtained in the second step by employing active cancellation in the RF domain. Among many $\mathrm{SIC}$ techniques in the RF domain proposed in the literature 
[3], [6], [7], the analog least mean square (ALMS) loop proposed in [7] is a promising structure due to its simplicity and ability to achieve significant SIC. As proved in [7]-[9], the transmitted signal properties have a noticeable impact on the performance of the ALMS loop. However, the analyses in these publications are only conducted for the random signals in FD communication systems. Therefore, two questions that could be raised are whether the ALMS loop works with deterministic signals, like in a GCW-SAR system, and if yes, how well it performs in this case.

To answer these questions, in this paper, we evaluate the performance of the ALMS loop in a GCW-SAR system with chirp signaling. A stationary analysis is applied to investigate the average convergence behavior of the loop by deriving the ensemble and time averaged weighting error function of the loop, which is the solution of an integral equation. It is proved that the ALMS loop can also work with the chirp signal in the GCW-SAR by a proper selection of the tap delay and number of taps in the loop. In particular, the tap delay of the loop must be selected according to the bandwidth of the chirp signal to avoid spectral overlapping. The number of taps in the loop is also constrained so that only one period of the autocorrelation function of the chirp signal is involved in the solution of the weighting error function. Simulation results show that about $45 \mathrm{~dB}$ SI suppression can be achieved with the ALMS loop under the selected simulation parameters. Combined with antenna separation, the total SI suppression requirement can be satisfied for a practical GCW-SAR system. Hence, the contributions of this paper are twofold. Firstly, it shows that the ALMS loop can work with both deterministic and random signals. Secondly, it proves that $70 \mathrm{~dB}$ of SIC can be obtained by using antenna separation and the ALMS loop in the RF domain with the given parameters. This level of SIC provides a reference for future researches on GCW-SAR.

The rest of this paper is organized as follows. In Section II, the GCW-SAR signal model and the ALMS loop filter are described. In Section III, we apply a stationary analysis to evaluate the behavior of the ALMS loop. In Section IV, simulations are conducted with two different scenarios of the SI channel to verify the theoretical analysis. Finally, Section $\mathrm{V}$ concludes the paper.

\section{System Description}

\section{A. GCW-SAR Signal Model}

Considering a GCW-SAR system, the transmitted signal $x(t)$ is defined as

$$
x(t)=\operatorname{Re}\left\{X(t) e^{j 2 \pi f_{c} t}\right\}
$$

where $X(t)$ is the low-pass equivalent of the transmitted signal and $f_{c}$ is the carrier frequency. In a conventional pulsed SAR system, a chirp signal pulse $P(t)$ is transmitted, and $P(t)$ can be expressed as

$$
P(t)=\operatorname{rect}\left(\frac{t}{T}\right) e^{j \pi k_{r} t^{2}}
$$

where $T$ is the pulse duration and $k_{r}$ is the chirp rate. However, in the GCW-SAR, the chirp signal $P(t)$ is transmitted periodically with the period of $T$, hence $X(t)$ is represented by $X(t)=\sum_{l=-\infty}^{\infty} V_{X} P(t-l T)$ where $V_{X}$ is the root mean square amplitude of the transmitted chirp signal, and it can be calculated by $V_{X}=\sqrt{\frac{1}{T} \int_{0}^{T}|X(t)|^{2} d t}$. The load is normalized to $1 \Omega$ so that the average power of $X(t)$ is $V_{X}^{2}$. At the input of the receiver, the received signal $r(t)$ is a combination of the SI $z(t)$, the reflected signal $s(t)$ from the target and the additive Gaussian noise $n(t)$, i.e.,

$$
r(t)=z(t)+s(t)+n(t) .
$$

The low-pass equivalents of these signals are denoted as $Z(t), S(t), N(t)$ respectively. The reflected signal $S(t)$ is a sum of a number of transmitted signals with different delays and attenuations, so that it can be modeled as

$$
S(t)=\int \sigma(\tau) X(t-\tau) d \tau
$$

where $\sigma(t)$ represents the reflection coefficients which are related to the radar cross sections of the surveyed area and their associated phases at the receiver antenna.

\section{B. ALMS Loop}

The GCW-SAR system is equipped with separated transmit and receive antennas, and they are located at a distance of $100 \mathrm{~mm}$. Hence at least $25 \mathrm{~dB}$ of SI attenuation can be achieved by the propagation loss [5]. In order to cancel the remaining SI, the ALMS loop proposed in [7] is employed. The architecture of the loop is presented in Fig. 1. The ALMS

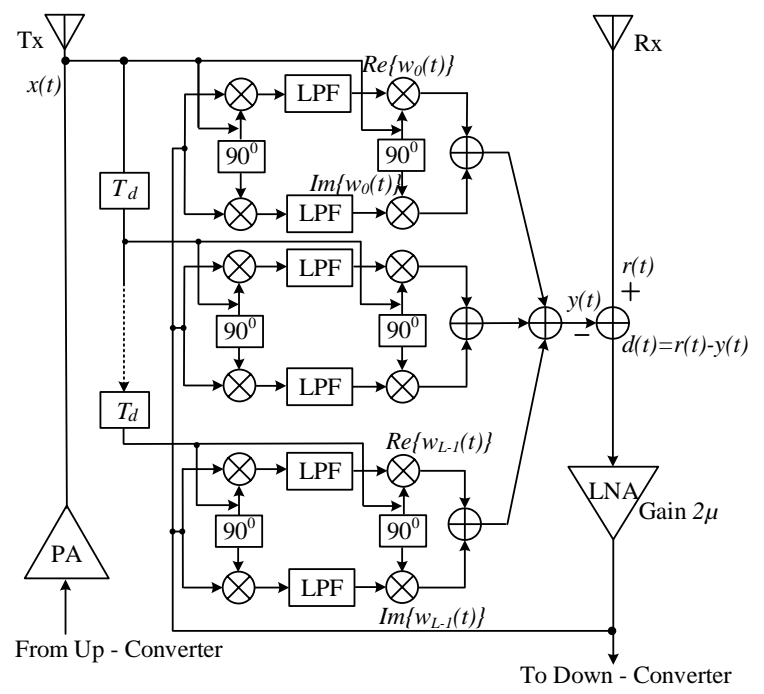

Fig. 1. The ALMS loop structure.

loop is an adaptive multi-tap filter which operates as follows. The transmitted signal $x(t)$ is passed into the ALMS filter including $L$-stage taps. At the $l$-th tap, the transmitted signal $x(t)$ is delayed by $l T_{d}$ before multiplied by the amplified residual signal $d(t)$. This product is filtered by a low-pass filter (LPF) to generate a weight coefficient $w_{l}(t)$ which will modify another version of the delayed signal $x(t)$. The outputs 
of all the taps are added together to obtain the cancellation signal $y(t)$. This cancellation signal is then used to subtract the SI $z(t)$ from the received signal $r(t)$. The involvement of the residual signal $d(t)$ to adaptively change the weighting coefficients forms a closed loop of the ALMS filter. As expressed in [7], the weighting coefficients $w_{l}(t)$ of the $l$-th tap can be expressed as

$$
\begin{array}{r}
w_{l}(t)=\frac{2 \mu \alpha}{K_{1} K_{2}} \int_{0}^{t} e^{-\alpha(t-\tau)}[r(\tau)-y(\tau)] \\
. X\left(\tau-l T_{d}\right) e^{j 2 \pi f_{c}\left(\tau-l T_{d}\right)} d \tau
\end{array}
$$

where $K_{1}$ and $K_{2}$ are the dimensional constants of the first and second multipliers in the loop, $\alpha=\frac{1}{R C}$ is the decay constant of the LPF, and $2 \mu$ is the gain of the low noise amplifier (LNA). From the architecture, the cancellation signal can be expressed as

$$
y(t)=\operatorname{Re}\left\{\sum_{l=0}^{L-1} w_{l}^{*} X\left(t-l T_{d}\right) e^{j 2 \pi f_{c}\left(t-l T_{d}\right)}\right\} .
$$

\section{STATIONARY ANALYSiS}

A stationary analysis is applied to evaluate the performance of the ALMS loop for the GCW-SAR system. This requires that both ensemble expectation and time averaging should be considered to derive the convergence behavior of the loop.

\section{A. Weighting Error Function}

The SI channel is modeled as a multi-tap filter so that the equivalent baseband version $Z(t)$ of the SI can be presented as $Z(t)=\sum_{l=0}^{L-1} h_{l}^{*} X\left(t-l T_{d}\right)$ where $L$ is the number of taps, and the tap delay of the SI channel is assumed to be equal to that in the ALMS loop for convenience at the moment. More general modeling of the SI channel will be discussed later in Section III. C. From (6), we can see that the performance of the ALMS loop can be represented by the error $u_{l}(t)$ between the $l$-th tap coefficient of the SI channel model $h_{l}$ and the corresponding weight of the adaptive filter, i.e.,

$$
u_{l}(t)=h_{l}-w_{l}(t) e^{j 2 \pi f_{c} T_{d} l} .
$$

With the assumption that the LPF bandwidth is very narrow relative to $f_{c}$, following the steps shown in $[7$, p. 4] to find $w_{l}(t)$, and substituting the result into (7), we have

$$
\begin{aligned}
u_{l}(t)= & h_{l}-\frac{\mu \alpha}{K_{1} K_{2}} \int_{0}^{t} e^{-\alpha(t-\tau)}\left[\sum_{l^{\prime}=0}^{L-1} u_{l^{\prime}}(\tau) X^{*}\left(\tau-l^{\prime} T_{d}\right)\right. \\
& \left.+S^{*}(\tau)+N^{*}(\tau)\right] X\left(\tau-l T_{d}\right) d \tau .
\end{aligned}
$$

Taking ensemble expectation of the above equation and assuming that the reflection coefficients are random and very small so that the ensemble expectation $E\left\{S^{*}(\tau) X\left(\tau-l T_{d}\right)\right\}$ is negligible in comparison with $E\left\{Z^{*}(\tau) X\left(\tau-l T_{d}\right)\right\}$, we have

$$
\begin{aligned}
\bar{u}_{l}(t) & =h_{l}-\frac{\mu \alpha}{K_{1} K_{2}} \int_{0}^{t} e^{-\alpha(t-\tau)} \\
& \cdot \sum_{l^{\prime}=0}^{L-1} \bar{u}_{l^{\prime}}(\tau) X^{*}\left(\tau-l^{\prime} T_{d}\right) X\left(\tau-l T_{d}\right) d \tau,
\end{aligned}
$$

where $\bar{u}_{l}(t)=E\left\{u_{l}(t)\right\}$. Eq. (5) implies that the ALMS loop starts at $t=0$. In general, the ALMS loop can start at any time instant $t_{0}$, thus (9) can be re-written as

$$
\begin{aligned}
\bar{u}_{l}\left(t+t_{0}\right)= & h_{l}-\frac{\mu \alpha}{K_{1} K_{2}} \int_{t_{0}}^{t+t_{0}} e^{-\alpha\left(t+t_{0}-\tau\right)} \sum_{l^{\prime}=0}^{L-1} \bar{u}_{l^{\prime}}(\tau) \\
& \cdot X^{*}\left(\tau-l^{\prime} T_{d}\right) X\left(\tau-l T_{d}\right) d \tau
\end{aligned}
$$

Defining $\tau^{\prime}=\tau-t_{0}$, we have

$$
\begin{aligned}
\bar{u}_{l}\left(t+t_{0}\right)= & h_{l}-\frac{\mu \alpha}{K_{1} K_{2}} \int_{0}^{t} e^{-\alpha\left(t-\tau^{\prime}\right)} \sum_{l^{\prime}=0}^{L-1} \bar{u}_{l^{\prime}}\left(\tau^{\prime}+t_{0}\right) \\
& \cdot X^{*}\left(\tau^{\prime}+t_{0}-l^{\prime} T_{d}\right) X\left(\tau^{\prime}+t_{0}-l T_{d}\right) d \tau^{\prime}
\end{aligned}
$$

Taking time average over one period $T$ of $\bar{u}_{l}\left(t+t_{0}\right)$ with respect to the starting time $t_{0}$, we obtain the ensemble and time averaged weighting error function $\overline{\bar{u}}_{l}(t)$ defined as

$$
\begin{aligned}
\overline{\bar{u}}_{l}(t)= & \frac{1}{T} \int_{0}^{T} \bar{u}_{l}\left(t+t_{0}\right) d t_{0} \\
= & h_{l}-\frac{\mu \alpha}{K_{1} K_{2} T} \int_{0}^{T} \int_{0}^{t} e^{-\alpha\left(t-\tau^{\prime}\right)} \sum_{l^{\prime}=0}^{L-1} \bar{u}_{l^{\prime}}\left(\tau^{\prime}+t_{0}\right) \\
& \cdot X^{*}\left(\tau^{\prime}+t_{0}-l^{\prime} T_{d}\right) X\left(\tau^{\prime}+t_{0}-l T_{d}\right) d \tau^{\prime} d t_{0} .
\end{aligned}
$$

Since $\alpha$ is very small, $\bar{u}_{l}(t)$ changes slowly and it can be seen as a constant in one period of $T$, i.e., $\bar{u}_{l}\left(t+t_{0}\right) \approx \overline{\bar{u}}_{l}(t)$, thus (12) can be written as

$$
\begin{aligned}
& \overline{\bar{u}}_{l}(t)=h_{l}-\mu \alpha \int_{0}^{t} e^{-\alpha\left(t-\tau^{\prime}\right)}\left[\sum_{l^{\prime}=0}^{L-1} \overline{\bar{u}}_{l^{\prime}}\left(\tau^{\prime}\right)\right. \\
& \left.\frac{1}{K_{1} K_{2} T} \int_{0}^{T} X^{*}\left(\tau^{\prime}+t_{0}-l^{\prime} T_{d}\right) X\left(\tau^{\prime}+t_{0}-l T_{d}\right) d t_{0}\right] d \tau^{\prime} \\
& =h_{l}-\mu \alpha \int_{0}^{t} e^{-\alpha\left(t-\tau^{\prime}\right)} \sum_{l^{\prime}=0}^{L-1} \overline{\bar{u}}_{l}\left(\tau^{\prime}\right) \Phi\left(\left(l-l^{\prime}\right) T_{d}\right) d \tau^{\prime}
\end{aligned}
$$

where $\Phi\left(\left(l-l^{\prime}\right) T_{d}\right)$ is the normalized autocorrelation function of the transmitted signal defined by

$$
\Phi(\tau)=\frac{1}{K_{1} K_{2} T} \int_{0}^{T} X^{*}(t) X(t-\tau) d t .
$$

To solve (13), we need to find the closed-form equation of the normalized autocorrelation function.

\section{B. Auto-Correlation Function of Transmitted Signal}

Since $X(t)$ is a periodical function with the period $T, \Phi(\tau)$ is also periodic with the period $T$. Hence, we can express the autocorrelation function as $\Phi(\tau)=\sum_{l=-\infty}^{\infty} A^{2} \Phi_{P}(\tau+l T)$ where $A^{2}=V_{X}^{2} / K_{1} K_{2}$ and $\Phi_{P}(\tau)$ is the autocorrelation function of $P(t)$ defined as:

$$
\begin{aligned}
\Phi_{P}(\tau) & =\frac{1}{T} \int_{0}^{T} P^{*}(t) P(t-\tau) d \tau \\
& =\frac{1}{T} \int_{0}^{T} \operatorname{rect}\left(\frac{t}{T}\right) \operatorname{rect}\left(\frac{t-\tau}{T}\right) e^{-j \pi k_{r} t^{2}} e^{j \pi k_{r}(t-\tau)^{2}} d t \\
& =\frac{1}{T} \int_{0}^{T} \operatorname{rect}\left(\frac{t}{T}\right) \operatorname{rect}\left(\frac{t-\tau}{T}\right) e^{j \pi k_{r}\left(-2 t \tau+\tau^{2}\right)} d t
\end{aligned}
$$

Obviously, $\Phi_{P}(\tau)=0$ if $\tau<-T$ and $\tau>T$. For $0 \leq \tau \leq T, \Phi_{P}(\tau)$ is found as

$$
\Phi_{P}(\tau)=\frac{T-|\tau|}{T} \operatorname{sinc}\left(\pi k_{r} \tau(T-|\tau|)\right) .
$$


$\Phi_{P}(\tau)$ is plotted in Fig. 2 .

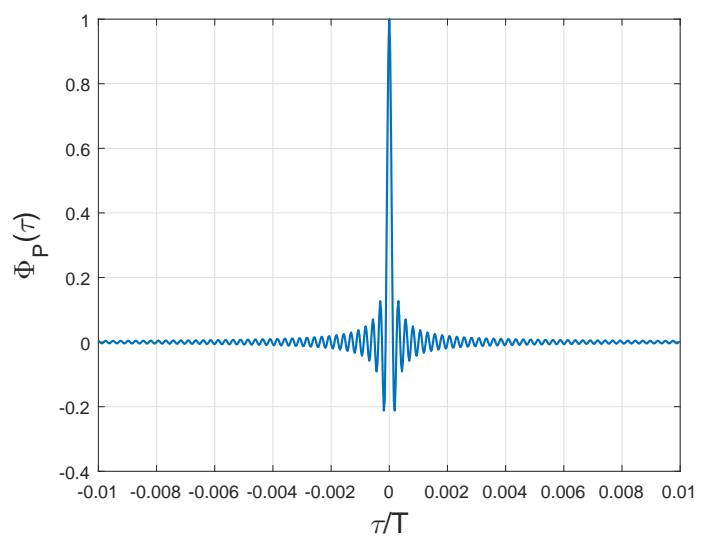

Fig. 2. The autocorrelation function of $P(t)$ with chirp rate $k_{r}=2.5 \times 10^{12}$.

\section{Solution of Weighting Error Function}

From $\Phi(\tau)$, (13) can be solved using the matrix form as in [7, pp. 7-8].

$$
\overline{\overline{\mathbf{u}}}(t)=\mathbf{h}-\mu \alpha \int_{0}^{t} e^{-\alpha(t-\tau)} \boldsymbol{\Phi} \overline{\overline{\mathbf{u}}}(\tau) d \tau
$$

where $\overline{\overline{\mathbf{u}}}(t)=\left[\begin{array}{lll}\overline{\bar{u}}_{0}(t) & \overline{\bar{u}}_{1}(t) & \cdots \overline{\bar{u}}_{L-1}(t)\end{array}\right]^{H}, \quad \mathbf{h}=$ $\left[\begin{array}{llll}h_{0} & h_{1} & \cdots & h_{L-1}\end{array}\right]^{H}$, and

$\boldsymbol{\Phi}=\left[\begin{array}{cccc}\Phi(0) & \Phi\left(-T_{d}\right) & \ldots & \Phi\left(-(L-1) T_{d}\right) \\ \Phi\left(T_{d}\right) & \Phi(0) & \cdots & \Phi\left(-(L-2) T_{d}\right) \\ \vdots & \vdots & \ddots & \vdots \\ \Phi\left((L-1) T_{d}\right) & \Phi\left((L-2) T_{d}\right) & \cdots & \Phi(0)\end{array}\right]$.

It can be seen from the matrix $\Phi$ that, each row of $\boldsymbol{\Phi}$ consists of $L$ samples of $\Phi(\tau)$ with sampling rate of $1 / T_{d}$. From the Nyquist theorem, in order to avoid spectral overlapping, it is required that $1 / T_{d} \geq 2 \frac{B}{2}$, where $B / 2$ is the maximum frequency of the chirp signal with the bandwidth $B\left(B=k_{r} T\right)$. Therefore, the tap delay of the ALMS loop must be selected as $T_{d}=\frac{1}{n B}$ where $n$ is integer, and $n \geq 1$. In addition, the number of taps $L$ also has to be limited so that $(L-1) T_{d} \leq T$. This condition ensures that only one period of $\Phi(\tau)$ is involved in deriving $\overline{\overline{\mathbf{u}}}(t)$. From these conditions, the solution for the weighting error functions derived in [7] can be applied to the chirp signal. In particular, the matrix $\boldsymbol{\Phi}$ is decomposed as $\boldsymbol{\Phi}=\mathbf{Q} \boldsymbol{\Lambda} \mathbf{Q}^{-1}$ where $\mathbf{Q}$ is the orthonormal modal matrix whose columns are the $L$ eigenvectors of $\boldsymbol{\Phi}$, and $\boldsymbol{\Lambda}$ is a diagonal matrix whose diagonal includes $L$ eigenvalues $\lambda_{l}$ of $\boldsymbol{\Phi}$. Noted that $\lambda_{l}$ are related to the loop gain $A^{2}$ as $\sum_{l=0}^{L-1} \lambda_{l}=L \Phi(0)=L A^{2}$. Using eigenvalue decomposition, $\overline{\overline{\mathbf{u}}}(t)$ is solved in [7] as

$$
\overline{\overline{\mathbf{u}}}(t)=\mathbf{Q} \operatorname{diag}\left\{\frac{1}{1+\mu \lambda_{l}}+\frac{\mu \lambda_{l}}{1+\mu \lambda_{l}} e^{-\left(1+\mu \lambda_{l}\right) \alpha t}\right\} \mathbf{Q}^{-1} \mathbf{h} .
$$

In order to evaluate the level of cancellation, the power of the residual SI is derived as

$$
\begin{aligned}
& P_{R I}(t)=\frac{1}{K 1 K 2} \bar{E}\left\{[z(t)-y(t)]^{2}\right\} \\
& =\frac{1}{K 1 K 2} \bar{E}\left\{\left[\operatorname{Re}\left\{Z(t)-\sum_{l=0}^{L-1}\left[h_{l}^{*}-u_{l}^{*}(t)\right] X\left(t-l T_{d}\right)\right\} e^{j 2 \pi f_{c} t}\right]^{2}\right\} \\
& =\frac{1}{2 K 1 K 2} \bar{E}\left\{\left|Z(t)-\sum_{l=0}^{L-1} h_{l}^{*} X\left(t-l T_{d}\right)\right|^{2}\right\} \\
& \left.\quad+\frac{1}{2 K 1 K 2} \bar{E}\left\{\mid \sum_{l=0}^{L-1} u_{l}^{*}(t) X\left(t-l T_{d}\right)\right] \mid\right\},
\end{aligned}
$$

where $\bar{E}\{$.$\} denotes combined ensemble and time averaging.$ In Section III. A, we have assumed that the SI channel is modeled as $L$ taps with the same tap delay as in the ALMS loop so that $Z(t)-\sum_{l=0}^{L-1} h_{l}^{*} X\left(t-l T_{d}\right)=0$. However, in practice the SI signal is better expressed by $Z(t)=\int_{-\infty}^{\infty} h^{*}(\tau) X(t-\tau) d \tau$ where $\mathrm{h}(\mathrm{t})$ is an arbitrary SI channel impulse response. In this case, the modeled tap coefficients $h_{l}$ can be obtained by minimizing the normalized modeling error expressed as

$$
\epsilon^{2}=\frac{1}{K 1 K 2} \bar{E}\left\{\left|Z(t)-\sum_{l=0}^{L-1} h_{l}^{*} X\left(t-l T_{d}\right)\right|^{2}\right\} .
$$

Applying the principal of orthogonality, that is $\bar{E}\{[Z(t)-$ $\left.\left.\sum_{l^{\prime}=0}^{L-1} h_{l}^{*} X\left(t-l^{\prime} T_{d}\right)\right] X\left(t-l T_{d}\right)\right\}=0$, the normalized modeling error is derived in [7] as

$$
\epsilon^{2}=\int_{-\infty}^{\infty} \int_{-\infty}^{\infty} h^{*}(\tau) h\left(\tau^{\prime}\right) \Phi\left(\tau-\tau^{\prime}\right) d \tau d \tau^{\prime}-\mathbf{h}^{H} \mathbf{\Phi} .
$$

Substituting (18) and (20) into (19), the normalized power of residual SI can be found as

$$
\begin{aligned}
P_{R I}(t)= & \frac{1}{2} \epsilon^{2}+\frac{1}{2} \mathbf{h}^{H} \mathbf{Q} \operatorname{diag}\left\{\frac{\lambda_{l}}{\left(1+\mu \lambda_{l}\right)^{2}}+\frac{2 \mu \lambda_{l}^{2}}{\left(1+\mu \lambda_{l}\right)^{2}} e^{-\left(1+\mu \lambda_{l}\right) \alpha t}\right. \\
& \left.+\frac{\mu^{2} \lambda_{l}^{3}}{\left(1+\mu \lambda_{l}\right)^{2}} e^{-2\left(1+\mu \lambda_{l}\right) \alpha t}-\bar{\lambda} e^{-2\left(1+\mu \lambda_{l}\right) \alpha t}\right\} \mathbf{Q}^{-1} \mathbf{h} .
\end{aligned}
$$

\section{Discussion}

1) When applied to a GCW-SAR system where the transmitted signal is deterministic, the ALMS loop behaves similarly as in a FD communication system where the transmitted signal is random. The weighting error functions $\overline{\overline{\mathbf{u}}}(t)$ converge to $\mathbf{Q} \operatorname{diag}\left\{\frac{1}{1+\mu \lambda_{l}}\right\} \mathbf{Q}^{-1} \mathbf{h}$ when $t \rightarrow \infty$. The convergence speed is driven by the loop gain $\mu A^{2}$ and the LPF parameter $\alpha$.

2) The above analyses are valid when the ALMS loop parameters satisfy two constraints of the tap delay $T_{d} \leq 1 / B$ and the number of taps $L$, i.e., $(L-$ 1) $T_{d} \leq T$. These conditions are essential for practical system design. 


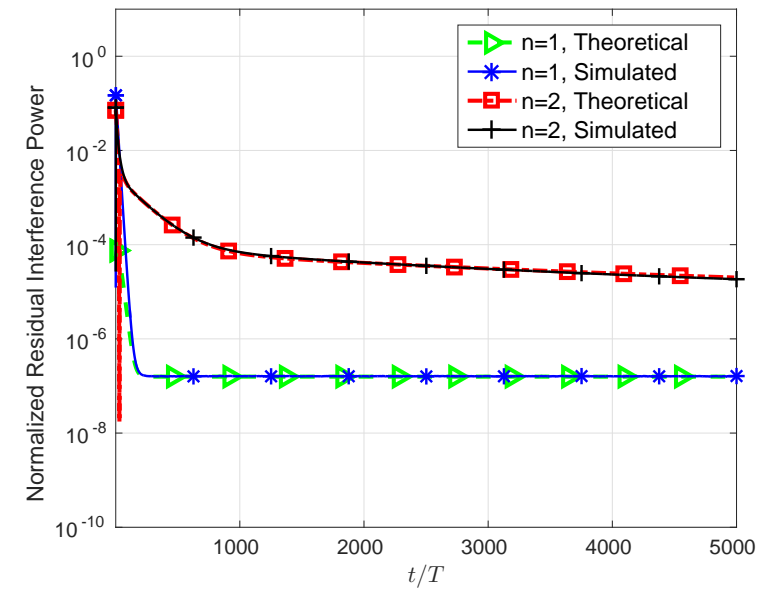

Fig. 3. The residual SI power in the first scenario for $T_{d}=1 / n B$, with $n=1,2$ and $L=4,8$ respectively.

\section{Simulation RESUlts}

The simulations are performed for the GCW-SAR with the following parameters. The transmitted chirp signal has the period $T$ of $40 \mu \mathrm{sec}$, and its power is $20 \mathrm{dBm}$ with $1 \mathrm{Ohm}$ load. The chirp rate is set with $k_{r}=2.5 \times 10^{12} \mathrm{~Hz}^{2}$ so that its bandwidth $B=k_{r} T=100 \mathrm{MHz}$. In the ALMS loop, the dimensional constants of the multipliers are set as $K_{1} K_{2}=0.001 V^{2}$ so that $A=10$. Another loop gain $\mu$ is selected as $\mu=10$. The LPF parameter $\alpha$ is set as $5 \times 10^{-5} \mathrm{sec}^{-1}$. The tap delay is chosen as $T_{d}=\frac{1}{n B}$ with $n=1,2$, and the number of taps are $L=4$ and $L=8$ respectively.

In the first scenario, the SI channel is selected with the delays being multiples of $T_{h}=1 / B$, for example $h(t)=10^{\frac{-25}{20}}\left\{\left[\frac{\sqrt{2}}{2}-0.5 j\right] \delta(t)-0.4 \delta\left(t-2 T_{h}\right)+0.3 \delta(t-\right.$ $\left.\left.4 T_{h}\right)\right\}$. It means that the ALMS loop has the same tap delay with the SI channel so that the modeling error is zero. The simulated and theoretical residual SI powers are presented in Fig. 3 for two cases of tap delay with $n=1,2$ and $L=4,8$ respectively. We can see that the level of cancellation given by the ALMS loop can be more than $60 \mathrm{~dB}$ in case of $T_{d}=1 / B$.

In the second scenario, the delays of the SI channel are fractional of $T_{h}$, for instance, $h(t)=10^{\frac{-25}{20}}\left\{\left[\frac{\sqrt{2}}{2}-0.5 j\right] \delta(t)-\right.$ $\left.0.4 \delta\left(t-0.9 T_{h}\right)+0.3 \delta\left(t-3.3 T_{h}\right)\right\}$. The simulation results are presented in Fig. 4. In this case, the modeling error has a significant impact on the performance of the ALMS loop. Particularly, the modeling error in case of $T_{d}=1 / B$ is up to $4 \times 10^{-3}$. Hence, the level of cancellation in this scenario is much lower than that in the first scenario for the same $T_{d}=1 / B$. However, with the finer tap delay in the loop ( $\left.T_{d}=1 / 2 B, L=8\right)$, the modeling error is reduced to $3.1525 \times 10^{-5}$. Hence, the level of cancellation is more than 45 $\mathrm{dB}$ which satisfies the requirement of SIC for the GCW-SAR system.

\section{CONCLUSION}

The stationary analysis is applied to investigate the behavior of the ALMS loop for the chirp signal in a GCW-SAR system.

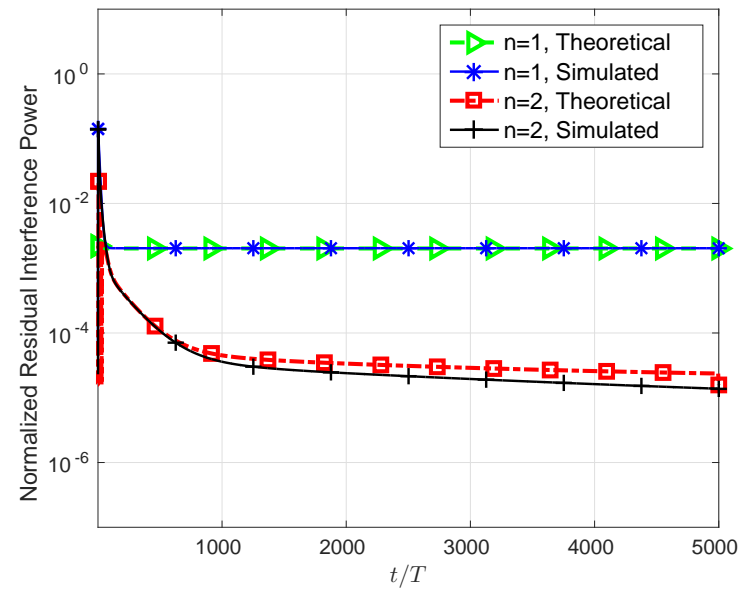

Fig. 4. The residual SI power in the second scenario for $T_{d}=1 / n B$ with $n=1,2$, and $L=4,8$ respectively.

Analysis and simulation results show that the ALMS loop works with the deterministic chirp signal in an imaging radar system as well as the random information bearing signal in a wireless communication system. It can be concluded that for the given loop gain, when the tap delay in the loop is properly selected, the level of cancellation more than $45 \mathrm{~dB}$ can be achieved. Together with the attenuation in the propagation domain by employing a distance between the transmit and receive antennas, the level of SI can be suppressed up to 70 $\mathrm{dB}$ at the input of the ADC. Future works about GCW-SAR can refer to this level of SIC to investigate the impacts of the residual SI.

\section{ACKNOWLEDGMENT}

This work was supported by the Australian Research Council (DP160101693).

\section{REFERENCES}

[1] Y. Nan, X. Huang, and Y. J. Guo, "A generalized continuous wave synthetic aperture radar," in Proc. 85th IEEE Vehicular Technology Conference (VTC Spring), June 2017, pp. 1-5.

[2] A. Currie and M. A. Brown, "Wide-swath SAR," IEE Proceedings F Radar and Signal Processing, vol. 139, no. 2, pp. 122-135, April 1992.

[3] Z. Zhang, K. Long, A. V. Vasilakos, and L. Hanzo, "Full-duplex wireless communications: Challenges, solutions, and future research directions," Proceedings of the IEEE, vol. 104, no. 7, pp. 1369-1409, July 2016.

[4] A. Meta, "Signal processing of FMCW synthetic aperture radar data," Ph.D. dissertation, Delft University of Technology, Netherlands, Aug. 2006.

[5] B. Debaillie et al., "Analog/RF solutions enabling compact full-duplex radios," IEEE J. Sel. Areas Commun, vol. 32, no. 9, pp. 1662-1673, June 2014.

[6] A. Kiayani et al., "Adaptive nonlinear RF cancellation for improved isolation in simultaneous transmit-receive systems," IEEE Trans. Microw. Theory Tech., vol. PP, no. 99, pp. 1-14, Jan 2018.

[7] X. Huang and Y. J. Guo, "Radio frequency self-interference cancellation with analog least mean-square loop," IEEE Trans. Microw. Theory Tech. vol. 65, no. 9, pp. 3336-3350, Sept 2017.

[8] A. T. Le, L. C. Tran, and X. Huang, "On performance of analog least mean square loop for self-interference cancellation in in-band full-duplex OFDM systems," in Proc. 85th IEEE Vehicular Technology Conference (VTC Spring), June 2017, pp. 1-5.

[9] — "Cyclostationary analysis of analog least mean square loop for selfinterference cancellation in in-band full-duplex systems," IEEE Commun. Lett, vol. 21, no. 12, pp. 2738-2741, Dec 2017. 